George Dantas de Azevedo'

Eduardo Caldas Costa ${ }^{2}$

Maria Thereza Albuquerque Barbosa

CABRAL MICUSSI ${ }^{2}$

JoCELINE CÁSSIA FEREZINI DE SÁ

Artigo de Revisão

\title{
Modificações do estilo de vida na síndrome dos ovários policísticos: papel do exercício físico e importância da abordagem multidisciplinar
}

\author{
Lifestyle modifications in the polycystic ovary syndrome: role of physical \\ exercise and importance of multidisciplinary approach
}

Palavras-chave

Exercício

Educação alimentar e nutricional Síndrome do ovário policístico Resistência à insulina Doenças cardiovasculares

Keywords

Exercise

Food and nutrition education

Polycystic ovaries syndrome Insulin resistance Cardiovascular diseases

\section{Resumo}

A síndrome dos ovários policísticos (SOP) é uma desordem endócrina heterogênea com prevalência de 5 a $10 \%$ nas mulheres em idade reprodutiva. Na SOP, há associação com vários fatores de risco para desenvolvimento de doença cardiovascular, como resistência à insulina, dislipidemia, diabetes mellitus, hipertensão arterial, disfunção endotelial, obesidade central, síndrome metabólica e marcadores pró-inflamatórios crônicos. A prática de exercício físico, juntamente com orientação nutricional, tem sido recomendada como estratégia de primeira linha no tratamento da oligomenorréia, hirsutismo, infertilidade e obesidade nas mulheres com SOP. Nesse sentido, o objetivo da presente revisão foi analisar o papel específico do exercício e/ou atividade física nas modificações da composição corporal, sistema cardiovascular, níveis plasmáticos bioquímicos e hormonais e função reprodutiva de mulheres com SOP.

\section{Abstract}

The polycystic ovaries syndrome (POS) is a heterogeneous endocrinal disorder prevalent in 5 to $10 \%$ of women in reproductive age. In POS, there is an association with risk factors linked to the development of cardiovascular disease such as insulin resistance, dislipidemia, diabetes mellitus, arterial hypertension, endothelial dysfunction, central obesity, metabolic syndrome and chronic pro-inflammatory markers. Physical exercise practice together with nutritional guidance have been recommended as first rate strategies in the treatment of oligomenorrhea, hirsutism, infertility and obesity in POS women. This way, the objective of the present review was to analyze the specific role played by exercise and/ or physical activity in changes of the body shape, in biochemical and hormonal plasmatic levels, and in the POS women's reproductive function. Universidade Federal do Rio Grande do Norte BR 101, Lagoa Nova - Campus Universitário $59078-970-$ Natal/RN Fone: (84) $3215-3431$ E-mail: georgedantas@uol.com.br
Grupo de Pesquisa Saúde da Mulher da Universidade Federal do Rio Grande do Norte - UFRN - Natal (RN), Brasil; Programa de Pós-graduação em Ciências da Saúde e Departamento de Morfologia da Universidade Federal do Rio Grande do Norte - UFRN Natal (RN), Brasil.

' Doutor, Professor Adjunto do Departamento de Morfologia e Orientador do Programa de Pós-graduação em Ciências da Saúde (PPGCSa) da Universidade Federal do Rio Grande do Norte - UFRN - Natal (RN), Brasil.

2 Pós-graduandos do Programa de Pós-graduação em Ciências da Saúde (PPGCSa) da Universidade Federal do Rio Grande do Norte - UFRN - Natal (RN), Brasil. 


\section{Introdução}

A síndrome dos ovários policísticos (SOP) é uma desordem endócrina heterogênea apresentada por 5 a $10 \%$ das mulheres em idade reprodutiva ${ }^{1,2}$. O diagnóstico dessa síndrome é firmado na presença de dois dos três fatores seguintes: anovulação crônica; sinais clínicos e/ou bioquímicos de hiperandrogenismo e presença de padrão ultra-sonográfico ovariano policístico ${ }^{3}$.

Além dos aspectos supracitados, é comum, na SOP, a presença de fatores de risco para desenvolvimento de doença cardiovascular (DCV), tais como resistência à insulina ${ }^{4}$, dislipidemia ${ }^{5}$, diabetes mellitus ${ }^{6}$, hipertensão arterial sistêmica ${ }^{6}$, disfunção endotelial ${ }^{7}$, obesidade central $^{8}$ e marcadores pró-inflamatórios crônicos ${ }^{9}$, além de baixa aptidão física ${ }^{10}$.

No tocante às repercussões cardiovasculares, tem sido demonstrada elevada prevalência de síndrome metabólica (SM) nas mulheres com SOP, inclusive no Brasil, conforme demonstrado recentemente. Evidencia-se elevada prevalência de SM em mulheres com SOP $(28,4 \%)$ na cidade de Natal, Rio Grande do Norte, especialmente no subgrupo de mulheres obesas $(52,3 \%)^{11}$. Uma vez que ainda não existem resultados de estudos com seguimento por tempo suficiente para avaliar a morbimortalidade por DCV nas mulheres com SOP, há necessidade de se desenvolver estudos de coorte especialmente desenhados para esse fim, com o intuito de verificar prováveis fatores de risco e possibilitar intervenções preventivas. Pelas características metodológicas inerentes a estudos dessa natureza, torna-se inviável a obtenção de resultados imediatos, motivo pelo qual se realizou previamente um estudo com metodologia de caso-controle, visando determinar a associação entre a ocorrência de irregularidade menstrual na idade reprodutiva e fatores de risco cardiovascular no período após a menopausa ${ }^{12}$.

No estudo citado, o antecedente de ciclos menstruais irregulares, que se constitui num dos principais indicadores de SOP, esteve associado ao aumento da razão de chance para ocorrência de hipertensão arterial [Odds Ratio ajustado $(\mathrm{OR})=2,4 ; 95 \%$ de intervalo de confiança $(\mathrm{IC})=1,395,41$ ], hipercolesterolemia $(\mathrm{OR}=2,32$; IC95\% $=1,174,59)$, hipertrigliceridemia $(\mathrm{OR}=2,09 ; \mathrm{IC} 95 \%=1,104,33) \mathrm{e}$ angioplastia coronariana $(\mathrm{OR}=6,69$; $\mathrm{IC} 95 \%=1,4331,21)$. Após ajuste para potenciais fatores de confusão também relacionados com o risco cardiovascular, tais como idade, índice de massa corpórea (IMC), antecedente familiar de DCV, tabagismo e inatividade física, o grupo de mulheres com irregularidade menstrual prévia apresentou maior chance para a ocorrência de algum fator de risco cardiovascular na pós-menopausa $(\mathrm{OR}=2,14 ; \mathrm{IC} 95 \%=1,024,48)$, quando comparadas àquelas com histórico de ciclos regulares prévios ${ }^{12}$.
Com base nesses resultados e considerando a relevância do tema em questão, torna-se necessária a abordagem preventiva dos fatores de risco identificáveis durante a vida reprodutiva da mulher, no sentido de minimizar a ocorrência futura de eventos mórbidos relacionados ao sistema cardiovascular, visto que estes constituem a principal causa de mortalidade na população feminina em nosso meio ${ }^{13}$.

$\mathrm{Na}$ atualidade, os objetivos do tratamento na SOP não se restringem à abordagem das repercussões reprodutivas, como infertilidade, anovulação e hirsutismo, sendo também direcionados para a promoção e prevenção da saúde cardiovascular. Nesse sentido, grande destaque tem sido dado às medidas não-farmacológicas, especialmente a orientação nutricional e prática regular de exercício e/ ou atividade física. Apesar das estratégias de tratamento de longa duração - mais efetivas para a SOP - não serem totalmente conhecidas ${ }^{14}$, parece ser fato que mudanças no estilo de vida, com modificações na dieta ${ }^{15-17}$, prática regular de exercício físico e perda de peso ${ }^{15-21}$ sejam mandatórias, somado à cessação do tabagismo, controle do estresse e consumo moderado de álcool ${ }^{15}$.

A prática regular de exercício físico tem sido recomendada como uma das estratégias de primeira linha no tratamento da obesidade, hiperandrogenismo e infertilidade das mulheres com SOP ${ }^{18,22}$. O exercício constitui-se num modulador positivo dos fatores de risco cardiovascular nessas mulheres ${ }^{23}$, tornando-se sua prática elemento indispensável no planejamento terapêutico ${ }^{15}$.

Apesar da relevância clínica, o exercício físico ainda tem sido pouco explorado como "ferramenta" terapêutica no tratamento da SOP, existindo apenas um discreto número de trabalhos científicos com esse foco específico. Além disso, não há aprofundamento acerca do tipo, intensidade, freqüência, duração e progressão dessa prática nas pacientes com SOP. Nesse sentido, o objetivo dessa revisão foi analisar o papel do exercício físico nas modificações da composição corporal, sistema cardiovascular, níveis bioquímicos e hormonais plasmáticos, assim como na função reprodutiva de mulheres com SOP.

Foi realizada revisão da literatura científica, por meio das bases de dados SciELO, Medline e Web of Science, selecionando-se estudos prospectivos de intervenção que envolveram a abordagem de exercício e atividade física em mulheres com SOP. A busca dos artigos foi realizada utilizando a palavra chave "polycystic ovary syndrome", que foi cruzada com os seguintes descritores: "exercise", "physical activity", "physical exercise", "exercise training", "physical training", "resistance training" e "lifestyle modification". Foram revisados os artigos de intervenção publicados até abril de 2008 e a seleção dos artigos incluiu como critério obrigatório uma duração mínima da intervenção de 12 semanas, independente do programa 
de exercícios físicos ter sido realizado isoladamente ou em associação com outra medida terapêutica, dietética e/ou medicamentosa.

A Tabela 1 sintetiza os principais estudos envolvendo a prática regular de exercício físico e seus efeitos sob diversos parâmetros de saúde em mulheres com SOP. Particularidades desses estudos serão discutidas a seguir.

\section{Efeitos sobre a composição corporal}

Mulheres com SOP e sobrepeso apresentam elevada prevalência de obesidade central, aspecto que se correlaciona diretamente com resistência aumentada à insulina $^{24}$. Huber-Huber-Buchholz, Carey e Norman ${ }^{18}$ mostraram que, após seis meses de exercício e dieta, mulheres obesas com SOP obtiveram alteração positiva na composição corporal, com redução da circunferência da cintura, melhora na sensibilidade à insulina, diminuição da insulina basal e redução do nível de hormônio luteinizante (LH), mesmo com baixo nível de perda da massa corporal total (2 a 5\%). Entretanto, esses achados só foram observados nas pacientes responsivas à intervenção, ou seja, as que se tornaram ovulatórias no decorrer e/ou no final da pesquisa.

A redistribuição da massa corporal gorda parece ser ainda mais importante do que sua perda, visto que a diminuição da obesidade central se acompanha de melhora na sensibilidade à insulina, com conseqüente impacto positivo na restauração da função ovariana ${ }^{18}$.

Confirmando os resultados do estudo anteriormente citado, Randeva et al. ${ }^{23}$ observaram resultados similares, após seis meses de prática regular de exercício aeróbico, com redução da relação cintura-quadril (RCQ), um parâmetro referente à obesidade central, mas sem evidenciarem alteração do IMC. Nesse estudo, foi utilizada a caminhada como forma de exercício; no primeiro trimestre, as voluntárias realizaram a atividade proposta três vezes por semana, somando 20 minutos por sessão; já no segundo trimestre de intervenção, houve incremento na duração e na freqüência dessa prática, sendo as caminhadas realizadas cinco vezes por semana, com acúmulo de aproximadamente 40 minutos por dia.

Ao se comparar os efeitos da mudança do estilo de vida com dieta e exercício físico (Grupo 1), utilização de metformina (Grupo 2) e combinação entre dieta, exercício e metformina (Grupo 3) em mulheres com SOP, observouse que, em todos os grupos, houve diminuição da massa corporal total, sendo a perda de peso mais significativa no Grupo $3^{14}$. Os autores deste estudo utilizaram 150 minutos de atividade física por semana como recomendação padrão para essas pacientes ${ }^{14}$.

Utilizando desenho metodológico mais apropriado, Bruner, Chad e Chizen ${ }^{17}$ desenvolveram o primeiro estudo com uma prescrição de exercício mais refinada para pacientes com SOP, constando da combinação de exercício aeróbico (caminhada e ciclismo estacionário) com exercícios resistidos (musculação). Os autores avaliaram o efeito desse programa associado ao aconselhamento nutricional, em comparação ao aconselhamento nutricional isolado. Após 12 semanas de intervenção, não foram observados efeitos significativos na perda de massa corporal e IMC. Entretanto, no tocante à circunferência da cintura, foi observada evolução semelhante em ambos os grupos, com diminuição da ordem de $5 \%$. Por outro lado, em relação ao somatório de dobras cutâneas, referência de percentual de gordura corporal total, somente o grupo que realizou exercício evoluiu de forma significativa, em comparação ao período pré-intervenção, com redução de $12 \%$. Isso leva a crer que as voluntárias que se exercitaram ganharam massa muscular e houve redução do percentual de gordura corporal total, apesar de não haver redução da massa corporal total e do IMC.

Tabela 1 - Resumo dos principais resultados de estudos de investigação sobre o efeito do exercício físico em mulheres com síndrome dos ovários policísticos

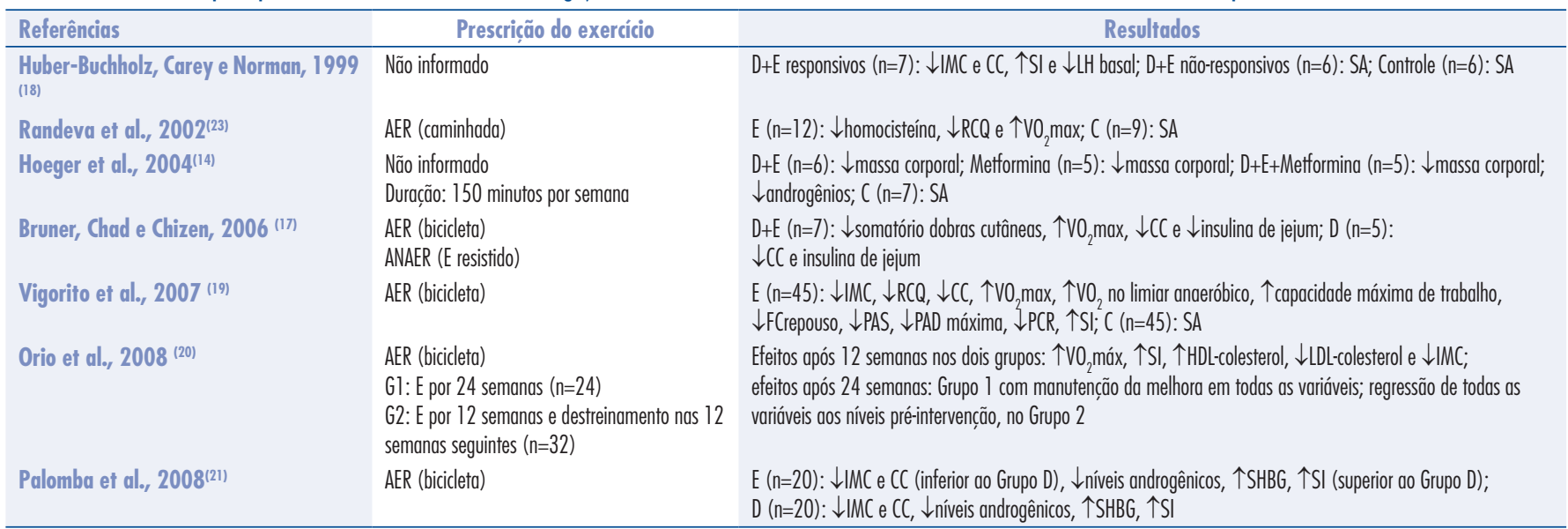

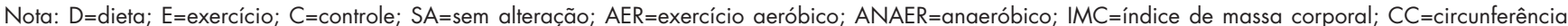
da cintura; $\mathrm{RCQ}=$ relação cintura-quadril; $\mathrm{Sl}=$ sensibilidade à insulina; $\mathrm{LH}=$ hormônio luteinizante; $\mathrm{VO}_{2}$ max=consumo máximo de oxigênio; $F C=$ freqüência cardíaca; $\mathrm{PAS}=$ pressão arterial sistólica; $\mathrm{PAD}=$ pressão arterial diastólica; $\mathrm{PCR}=$ proteína $\mathrm{C}$ reativa; $\mathrm{SHBG=globulina}$ de ligação dos hormônios sexuais 
Ainda nesse estudo, um achado interessante no que diz respeito à taxa metabólica de repouso (TMR): o grupo que realizou exercício obteve aumento de $9 \%$ nessa variável, ao contrário do grupo controle, que teve redução de $1 \%$. Apesar da ausência de significância ${ }^{17}$, esse dado não deve ser ignorado do ponto de vista clínico, principalmente por se tratar de aspecto relevante na manutenção da massa corporal.

Outros estudos analisaram intensidades de exercício e parâmetros diferentes. Observou-se que, após três meses de um programa de exercício aeróbico, numa freqüência de três vezes por semana, 30 minutos de duração e intensidade entre 60 a $70 \%$ do $\mathrm{VO}_{2} \max$, houve redução do IMC, circunferência da cintura e RCQ ${ }^{19}$. Em outro estudo, em que se empregou prescrição de exercício semelhante no que se refere ao tipo, intensidade, duração e freqüência, foram analisados os efeitos do treinamento físico seguido de destreinamento nas pacientes com $\mathrm{SOP}^{20}$. A amostra foi dividida em dois grupos: o Grupo 1 realizou 24 semanas de um programa de exercício aeróbico moderado, e o Grupo 2 realizou um período de 12 semanas do mesmo protocolo, seguido por 12 semanas sem exercícios, retornando ao estado sedentário. Em ambos os grupos, houve semelhante diminuição do IMC, circunferência da cintura e RCQ, após 12 semanas de intervenção. Por outro lado, na segunda avaliação, realizada 24 semanas após o início do estudo, o grupo que manteve o programa de exercícios físicos continuou evoluindo favoravelmente em todos os parâmetros descritos, ao contrário do grupo que sofreu destreinamento, o qual involuiu em todas as variáveis, retornando ao perfil do período pré-intervenção ${ }^{20}$.

Apesar das inconsistências existentes em relação ao tipo, intensidade e freqüência dos programas de exercícios físicos a serem utilizados em mulheres jovens com $\mathrm{SOP}$, os resultados dos estudos disponíveis indicam que a prática regular de exercício físico deve ser incentivada e mantida por um período prolongado nas mulheres com SOP, tendo em vista que um curto período de cessação da atividade física pode provocar anulação dos benefícios previamente alcançados ${ }^{20}$.

De forma dicotomizada, Palomba et al. ${ }^{21}$ compararam os efeitos isolados da dieta alimentar (Grupo 1) e da prática de exercício físico (Grupo 2) na função reprodutiva de mulheres com SOP, incluindo também avaliação de parâmetros relativos à composição corporal. Nesse sentido, também utilizaram a mesma metodologia de treinamento proposta por Vigorito et al. ${ }^{19}$. Os autores verificaram mudanças significativas em ambas as intervenções no que diz respeito à massa corporal, IMC, circunferência da cintura e RCQ, mas apenas nas pacientes consideradas responsivas às intervenções, ou seja, aquelas que se tornaram ovulatórias no decorrer do estudo.

\section{Parâmetros cardiovasculares}

A SOP representa um complexo modelo biológico no que concerne à relação entre perfil hormonal e risco cardiovascular. Nas mulheres com SOP, a combinação entre resistência à insulina e um fenótipo de sedentarismo gera um perfil de risco cardiovascular ainda mais desfavorável, com tendência a aumento do IMC, baixa capacidade cardiopulmonar, redução da aptidão física e estado pró-inflamatório crônico aumentado ${ }^{19}$. Logo, torna-se importante conhecer o papel do exercício na modulação desses aspectos.

O trabalho de Randeva et al. ${ }^{23}$ revelou diminuição na concentração de homocisteína plasmática em mulheres jovens com sobrepeso e obesas com SOP, que aderiram ao programa de exercício proposto, resultado que não foi evidenciado no grupo controle. Um aspecto clinicamente importante nesse estudo é que as pacientes que apresentaram os maiores níveis basais de homocisteína foram as que mais se beneficiaram da prática de exercício físico, no que diz respeito à melhora deste parâmetro. Portanto, considerando que as mulheres com SOP apresentam níveis aumentados de homocisteína plasmática ${ }^{25}$, o exercício torna-se também uma ferramenta terapêutica importante nesse sentido, uma vez que níveis elevados de homocisteína constituem importante fator de risco cardiovascular ${ }^{26}$.

Adicionalmente à redução dos níveis de homocisteína plasmática, o estudo de Randeva et al..$^{23}$ parece ter sido o primeiro a demonstrar o efeito positivo de um programa regular de exercício em parâmetros de funcionalidade cardiovascular, observando melhora no consumo máximo de oxigênio ( $\mathrm{VO}_{2}$ max) em pacientes com SOP. Posteriormente, Bruner Chad e Chizen ${ }^{17}$ também notificaram incremento substancial do $\mathrm{VO}_{2}$ max no grupo que combinou prática regular de exercício com aconselhamento nutricional, sendo esse aumento da ordem de $42 \%$ após 12 semanas de treinamento. As voluntárias que receberam apenas aconselhamento nutricional não obtiveram melhora significante nesse parâmetro.

Esses resultados são significativos, pois destacam a influência do exercício físico sobre esse marcador de extrema relevância clínica $\left(\mathrm{VO}_{2} \max \right)$ e que se correlaciona negativamente com mortalidade cardiovascular e, indiretamente, com todas as demais causas ${ }^{27-31}$. $\mathrm{O}$ aumento do $\mathrm{VO}_{2}$ max observado após treinamento aeróbico nas mulheres com SOP parece se relacionar com redução do IMC, circunferência da cintura, RCQ e área sob a curva de insulina, além de aumento na relação glicose-insulina ${ }^{19}$. Esses achados também confirmam estudos previamente publicados, que demonstraram uma importante correlação negativa entre resistência à insulina $\mathrm{eVO}_{2} \max ^{32,33}$.

Nessa mesma linha de investigação, observou-se melhora na capacidade máxima de trabalho, aumento do 
consumo de oxigênio no limiar anaeróbico (LA), diminuição da freqüência cardíaca (FC) de repouso, da pressão arterial sistólica (PAS) de repouso, da pressão arterial diastólica (PAD) máxima e do nível basal de proteína $\mathrm{C}$ reativa após três meses de prática regular de exercício aeróbico ${ }^{19}$. Outros achados foram o incremento no nível de atividade física e o gasto calórico diário das pacientes, incluindo atividades recreacionais e domésticas, tendo esses aspectos se correlacionado positivamente com melhora no $\mathrm{VO}_{2} \max$, área sob a curva de insulina e relação glicose-insulina.

No estudo anteriormente citado de Orio et al. ${ }^{20}$, foi observado aumento do $\mathrm{VO}_{2}$ max, carga máxima de esforço e consumo de oxigênio no LA, após 12 semanas de treinamento aeróbico de moderada intensidade - efeitos que foram conservados apenas no grupo que manteve a prática de exercícios físicos. Por outro lado, as pacientes que sofreram destreinamento exibiram involução em todas as variáveis supracitadas, voltando para o nível préintervenção. Esse contexto denota que um maior tempo de engajamento nesse tipo de intervenção (prática de exercício regular) se relaciona com modificações ascendentes em parâmetros relacionados à saúde cardiovascular em mulheres com SOP.

\section{Parâmetros bioquímicos e hormonais}

Há evidências de que a redução da massa corporal em mulheres com SOP é efetiva para melhora da sensibilidade à insulina ${ }^{14,17,18}$. Sabendo que a resistência à insulina parece exercer um papel chave na fisiopatologia da SOP, torna-se interessante averiguar se o exercício per se é uma estratégia eficaz para produzir efeito semelhante à perda de massa corporal ${ }^{17}$.

De forma preliminar, verificou-se que a resistência à insulina apresentou relação direta com a circunferência da cintura, obesidade central e nível de insulina basal em mulheres com $\mathrm{SOP}^{18}$, embora este estudo tivesse incluído pequeno número de sujeitos. Hoeger et al. ${ }^{14}$ demonstraram que a combinação de metformina, dieta e prática regular de exercício foi eficiente na diminuição de 4,5\% nos níveis de testosterona plasmática e de 15,2\% nos de androgênios livres (FAI). Tais resultados não foram verificados no grupo que fez uso isolado de metformina, nem no qual se introduziu apenas mudanças de estilo de vida, com a dieta e prática regular de exercício. A redução dos níveis de testosterona plasmática, assim como os de FAI, e o aumento de SHBG (do inglês sex hormonebinding globulin) foram relatados em outro estudo, tanto em um grupo que passou a realizar exercícios físicos regulares quanto no grupo submetido à dieta alimentar hiperprotéica e hipocalórica ${ }^{21}$. Entretanto, o grupo que adotou apenas a dieta apresentou menor nível de FAI e maior nível SHBG. Além disso, os índices de sulfato de dehidroepiandrosterona (DHEA-S) e androstenediona plasmática só foram reduzidos no grupo que se submeteu a dieta. Estudos adicionais são necessários para esclarecer o impacto dos exercícios físicos sobre os parâmetros clínicos e hormonais de hiperandrogenismo.

Por favorecer a utilização periférica da glicose e melhorar a sensibilidade à insulina, a prática regular de exercícios físicos está associada à diminuição nos níveis de insulina de jejum em pacientes com SOP ${ }^{17}$. Vigorito et al. ${ }^{19}$ demonstraram modificações positivas no nível de insulina de jejum, na área sob a curva de insulina e na relação glicose-insulina, após três meses de prática de exercício aeróbico de moderada intensidade. Os mesmos resultados foram encontrados no estudo publicado por Orio et al. ${ }^{20}$, tendo o grupo que sofreu destreinamento apresentado o mesmo comportamento de involução após cessação do programa de exercício.

Em relação ao perfil lipídico, a prática regular de exercícios físicos é capaz de promover diminuição significante nos níveis de LDL-colesterol e aumento do HDL-colesterol, na dependência da duração do treinamento ${ }^{20}$. Logo, parece ser fato que a realização de exercício físico regular em pacientes com SOP promove impacto positivo sobre diversos parâmetros bioquímicos e hormonais, com destaque para a melhora da sensibilidade periférica à insulina.

\section{Função reprodutiva}

Um dos principais objetivos no tratamento da SOP é a restauração da função ovulatória. Em uma investigação na qual este desfecho foi analisado, pacientes que realizaram seis meses de prática regular de exercício físico associada à dieta apresentaram melhora do perfil ovulatório, tendo duas dessas pacientes engravidado durante o período experimental ${ }^{18}$. Os autores associam essas mudanças positivas principalmente ao aumento na sensibilidade à insulina, que precedeu a ovulação nas pacientes que melhoraram esse aspecto.

Hoeger et al. ${ }^{14}$ observaram uma tendência clínica a maior freqüência de ovulações regulares, documentadas pela excreção urinária de pregnanediol, nos grupos experimentais (dieta e exercício, metformina e combinação dos três), em comparação ao grupo controle, apesar da falta de significância nesta comparação. Nesse mesmo estudo, uma análise mais detalhada demonstrou que o grupo de mulheres que perdeu $3 \%$ ou mais de massa corporal apresentou nove vezes mais chance de ovular regularmente, quando comparado ao grupo que não atingiu essa perda relativa mínima de massa corporal (OR=8,9; IC95\%=1,2-64,6). Associando essa abordagem ao uso de metformina, esse índice sobe para 16 vezes $(\mathrm{OR}=16,1$; IC95\%=4,3-64,2). Tais evidências associadas com observações de outros 
estudos ${ }^{19}$ são significativas para demonstrar o impacto positivo que tem o exercício físico (seja isolado ou em associação com dieta e/ou metformina) sobre a melhora da função reprodutiva de mulheres com SOP.

$\mathrm{O}$ estudo de Palomba et al. ${ }^{21}$ investigou de forma ainda mais específica os efeitos de um programa de exercício físico sobre os parâmetros reprodutivos de mulheres com SOP, analisando também os mesmos dados em um grupo tratado apenas com dieta. Os resultados mostraram aumento da ciclicidade menstrual em ambos os grupos, sem diferença. Entretanto, a freqüência de menstruações, ou seja, razão de fluxos menstruais observados pelo número de fluxos menstruais esperados, foi significantemente maior no grupo que realizou exercício (26,2 versus $15,3 \%)$. Nesse mesmo estudo, após 24 semanas, tanto o índice de ovulação (número de ciclos ovulatórios/número de ciclos observados) quanto o índice de ovulação cumulativo (pacientes ovulatórias/número de pacientes) foi maior no grupo que realizou exercício físico em comparação ao grupo que fez apenas a dieta. Além disso, houve tendência a um maior índice de gravidez (número de gravidezes/número de ciclos observados) e índice de gravidez cumulativo (número de pacientes grávidas/número de pacientes) no grupo que realizou exercício. Esses achados, mais uma vez, parecem correlacionar positivamente a prática regular de exercício físico com melhora na função reprodutiva.

\section{Conclusão}

A prática regular de exercício físico em mulheres com SOP tem demonstrado importância terapêutica relevante, uma vez que as evidências indicam resultados positivos dessa modalidade nos aspectos relacionados à composição corporal, parâmetros metabólicos, cardiovasculares e hormonais, além da função reprodutiva.
Entretanto, o conhecimento é ainda incipiente no que diz respeito à relação dose-resposta para obtenção desses benefícios, assim como no tocante ao tipo, intensidade, duração, freqüência e progressão dos exercícios. O que parece ser fato é a grande utilização dos exercícios aeróbicos como base da prescrição dos programas de treinamento para mulheres com SOP. A utilização dos exercícios resistidos encontra-se ainda nebulosa nesse sentido, com ausência de trabalhos dando ênfase a essa modalidade específica de prática. Em nosso grupo de pesquisa, encontra-se em andamento um estudo especificamente desenhado para essa finalidade, de forma que, num futuro próximo, pretende-se dispor de subsídios para orientar decisões clínicas nesse sentido, tendo por base evidências científicas mais adequadas ao nosso perfil epidemiológico.

Para que se possam obter prognósticos favoráveis, as evidências atuais sugerem que a prática de exercício físico deve ser estimulada e mantida por longo prazo nas mulheres com SOP, uma vez que sua suspensão, mesmo que por curtos períodos, é capaz de gerar perdas relevantes, principalmente em relação aos aspectos metabólicos e cardiovasculares.

Por fim, ainda existem muitos fatores de confundimento nos estudos, os quais limitam conclusões mais concretas sobre o papel específico do exercício físico no tratamento da SOP. O que está claro é seu papel potencializador das estratégias terapêuticas dietéticas e/ou medicamentosas, visando à melhoria nas funções hormonal, metabólica, cardiovascular e reprodutiva.

\section{Agradecimentos}

Agradecemos as colaborações da Doutora Elvira Maria Mafaldo Soares e da Professora Doutora Técia Maria de Oliveira Maranhão na revisão final desse artigo.

\section{Referências}

1. Norman RJ, Dewailly D, Legro RS, Hickey TE. Polycystic ovary syndrome. Lancet. 2007;370(9588):685-97.

2. Kelly CJ, Connell JM, Cameron IT, Gould GW, Lyall H. The long term health consequences of polycystic ovary syndrome. BJOG. 2000; 107(11):1327-38

3. The Rotterdam ESHRE/ASRM-Sponsored. PCOS Consensus Workshop Group. Revised 2003 consensus on diagnostic criteria and long-term health risks related to polycystic ovary syndrome. Fertil Steril. 2004;81(1):19-25.

4. Park KH, Kim JY, Ahn CW, Song YD, Lim SK, Lee HC. Polycystic ovarian syndrome (PCOS) and insulin resistance. Int J Gynaecol Obstet. $2001 ; 74(3): 261-7$.

5. Cussons AV, Stuckey BGA, Watts GF. Cardiovascular disease in the polycystic ovary syndrome: new insights and perspectives. Atherosclerosis. 2006;185(2):227-39.
6. Cibula D, Cífková F, Fanta M, Poledne R, Zivny J, Skibová J. Increased risk of non-insulin dependent diabetes mellitus, arterial hypertension and coronary artery disease in perimenopausal women with a history of the polycystic ovary syndrome. Hum Reprod. 2000; 15(4):785-9.

7. Paradisi G, Steinberg HO, Hempfling A, Cronin J, Hook G, Shepard $M K$, et al. Polycystic ovary syndrome is associated with endothelial dysfunction. Circulation. 2001;103(10):1410-5.

8. Lord J, Thomas R, Fox B, Acharya U, Wilkin T. The central issue? Visceral fat mass is a good marker of insulin resistance and metabolic disturbance in women with polycystic ovary syndrome. BJOG. 2006; 1 13(10):1203-9.

9. Kelly CC, Lyall H, Petrie JR, Gould GW, Connell JM, Sattar N. Low grade chronic inflammation in women with polycystic ovarian syndrome. J Clin Endocrinol Metab. 2001;86(6):2453-5. 
10. Orio F, Giallauria F, Palomba S, Cascella T, Manguso F, Vuolo L, et al. Cardiopulmonary impairment in young women with polycystic ovary syndrome. J Clin Endocrinol Metab. 2006;91(8):2967-71.

11. Soares EM, Azevedo GD, Gadelha RG, Lemos TM, Maranhão TM. Prevalence of the metabolic syndrome and its components in Brazilian women with polycystic ovary syndrome. Fertil Steril. 2008;89(3):649-55.

12. Azevedo GD, Duarte JM, Souza MO, Costa-E-Silva TD, Soares EM, Maranhão TM. Irregularidade do ciclo menstrual no menacme como marcador para fatores de risco cardiovasculares na pós-menopausa. Arq Bras Endocrinol Metabol. 2006;50(5):876-83.

13. Soares EMM, Soares GM, Silva Filho JG, Melo MCL, Azevedo GD, Maranhão TMO. Causas de óbitos femininos no Rio Grande do Norte (RN) em 2002 e 2003 [videocassete]. In: Anais do $51^{\circ}$ Congresso Brasileiro de Ginecologia e Obstetrícia; 2005 nov 2226; Rio de Janeiro, Brasil. Rio de Janeiro: TV MED; 2005.

14. Hoeger KM, Kochman L, Wixom N, Craig K, Miller RK, Guzick DS. A randomized, 48-week, placebo-controlled trial of intensive lifestyle modification and/or metformin therapy in overweight women with polycystic ovary syndrome: a pilot study. Fertil Steril. 2004;82(2):421-9.

15. Norman RJ, Davies M, Lord J, Moran LJ. The role of lifestyle modification in polycystic ovary syndrome. Trends Endocrinol Metab. 2002;13(6):251-7.

16. Moran L, Brinkworth G, Noakes M, Norman RJ. Effects of lifestyle modification in polycystic ovarian syndrome. Reprod Biomed Online. 2006; 12(5):569-78

17. Bruner B, Chad K, Chizen D. Effects of exercise and nutritional counseling in women with polycystic ovary syndrome. Appl Physiol Nutr Metab. 2006;31 (4):384-91.

18. Huber-Buchholz MM, Carey DG, Norman RJ. Restoration of reproductive potential by lifestyle modification in obese polycystic ovary syndrome: role of insulin sensitivity and luteinizing hormone. J Clin Endocrinol Metab. 1999;84(4):1470-4.

19. Vigorito C, Giallauria F, Palomba S, Cascella T, Manguso F, Lucci $R$, et al. Beneficial effects of a three-month structured exercise training program on cardiopulmonary functional capacity in young women with polycystic ovary syndrome. J Clin Endocrinol Metab. 2007;92(4): 1379-84.

20. Orio F, Giallauria F, Palomba S, Manguso F, Orio M, Tafuri D, et al. Metabolic and cardiopulmonary effects of detraining after a structured exercise training programme in young PCOS women. Clin Endocrinol (Oxf). 2008;68(6):976-81.

21. Palomba S, Giallauria F, Falbo A, Russo T, Oppedisano R, Tolino A, et al. Structured exercise training programme versus hypocaloric hyperproteic diet in obese polycystic ovary syndrome patients with anovulatory infertility: a 24-week pilot study. Hum Reprod. 2008;23(3):642-50.
22. Cussons AJ, Stuckey BG, Walsh JP, Burke V, Norman RJ. Polycystic ovarian syndrome: marked differences between endocrinologists and gynaecologists in diagnosis and management. Clin Endocrinol (Oxf). 2005;62(3):289-95.

23. Randeva HS, Lewandowski KC, Drzewoski J, Brooke-Wavell K, $\mathrm{O}^{\prime}$ Callaghan C, Czupryniak L, et al. Exercise decreases plasma total homocysteine in overweight young women with polycystic ovary syndrome. J Clin Endocrinol Metab. 2002;87(10): 4496-501.

24. Cascella T, Palomba S, De Sio I, Manguso F, Giallauria F, De Simone $B$, et al. Visceral fat is associated with cardiovascular risk in women with polycystic ovary syndrome. Hum Reprod. 2008;23(1): 153-9.

25. Yarali H, Yildirir A, Aybar F, Kabakçi G, Bükülmez O, Akgül E, et al. Diastolic dysfunction and increased serum homocysteine concentrations may contribute to increased cardiovascular risk in patients with polycystic ovary syndrome. Fertil Steril. 2001;76(3):51 1-6.

26. Boushey CJ, Beresford SA, Omenn GS, Motulsky KG. A quantitative assessment of plasma homocysteine as a risk factor for vascular disease: probable benefits of increasing folic acid intakes. JAMA. 1995;274(13):1049-57.

27. Blair SN, Kohl HW 3rd, Barlow CE, Paffenbarger Jr RS, Gibbons LW, Macera CA. Changes in physical fitness and all-cause mortality. A prospective study of healthy and unhealthy men. JAMA. 1995;273(14):1093-8.

28. Erikssen G, Liestøl K, Biørnholt J, Thaulow E, Sandvik L, Erikssen J. Changes in physical fitness and changes in mortality. Lancet. 1998;352(9130):759-62

29. Lamonte M, Eisenman PA, Adams TD, Shults BB, Ainsworth BE, Yanowitz FG. Cardiorespiratory fitness and coronary heart disease risk factors: the LDS Hospital Fitness Institute Cohort. Circulation. 2000; 102(14): 1623-8.

30. Laukkanen JA, Lakka TA, Rauramaa R, Kuhanen R, Venalainen JM, Salonen $\mathrm{R}$, et al. Cardiovascular fitness as a predictor of mortality in men. Arch Intern Med. 2001;161(6):825-31.

31. Kavanagh T, Mertens DJ, Hamm LF, Beyene J, Kennedy J, Corey $\mathrm{P}$, et al. Prediction of long-term prognosis in 12.169 men referred for cardiac rehabilitation. Circulation. 2002;106(6): 666-71.

32. Seibaek M, Vestergaard H, Burchardt $H$, Sloth $C$, Torp-Pedersen $C$, Nielsen SL, et al. Insulin resistance and maximal oxygen uptake. Clin Cardiol. 2003;26(1 1):515-20.

33. Sirikul B, Gower BA, Hunter GR, Larson-Meyer DE, Newcomer BR. Relationship between insulin sensitivity and in vivo mitochondrial function in skeletal muscle. Am J Physiol Endocrinol Metab. 2006;291(4):E724-8. 